\author{
Military Technical College \\ Kobry El-Kobbah, \\ Cairo, Egypt
}

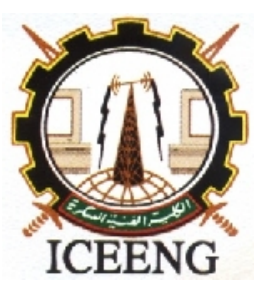

\author{
$8^{\text {th }}$ International Conference \\ on Electrical Engineering \\ ICEENG 2012
}

\title{
Modeling and Measurements of Laser Spot Position Determination Circuit with a Quad Detector
}

\author{
By \\ Mohamed F. Heweage * \\ Samy A. Ghoniemy * \\ Ayman M. Mokhtar* \\ Mekheimer A. Soliman * \\ Ahmed Eldamarawy*
}

\section{Abstract:}

In this paper we are interested in modeling, simulation, and measurement of laser spot position determination circuit (LSPDC) with a quadrant detector (QD). Photodiode is modeled using its differential equations then implemented using ORCAD -pspise. Starting with the output of the detector, the circuit modeling and simulation for the preamplifier, post amplifier, ADC and microcontroller card were performed. PCB card implementation was performed and integrated with the QD and the necessary optical system components. The implementation of the full LSPDC with the detector is performed. General system for testing the spot LSPDC was performed with a laser source simulating the incident radiation and a mechanical system to enable adjusting different angles. Good agreement was achieved between the measured data and the adjusted angles with a small error attributed to the error in the adjusted mechanical system and the stability of the used laser source.

\section{Keywords:}

Photodiode modeling, Quad detector, spot position measurements

\section{Introduction:}

Laser tracking systems (LTS) are used for detecting and tracking the targets by obtaining the position information of these targets. The main parts of the LTS are the optical system for collimating the reflected beam from the target. The position sensitive

* Egyptian Armed Forces 
detector which converts the incident laser spot to its corresponding photocurrent, and the electronic circuits that, filter and convert the photocurrent to its corresponding voltage. The position sensitive diode (PSD) is the core component in the laser tracker. Laser tracking systems (LTS) are used for detecting and tracking the targets by obtaining the position information of these targets. The main parts of the LTS are the optical system for collimating the reflected beam from the target, the position sensitive detector which converts the incident laser spot to its corresponding photocurrent, and the electronic circuits that, filter and convert the photocurrent to its corresponding voltage. The position sensitive diode (PSD) is the core component in the laser tracker. A PSD is a continuous silicon photodetector used for optical position sensing and basically consists of a uniform resistive layer, which is formed on a silicon substrate. A pair of electrodes are formed at the ends of the resistive layer from which photo-currents are measured. These photo-currents are generated as a result of the incident radiation effect and their magnitude is relative to the distance of the electrode to the center of the beam spot on the sensor's active area. The photo-currents are typically amplified and converted to form the measured voltage signal used by the control system. PSDs can be divided into two general groups: segmented PSDs and lateral effect PSDs in this paper we will consider segmented PSDs which are quadrate PSD (QD), each is divided into four segments, separated by a small gap, called the dead region. In QD the position is extracted by comparing each segment's photocurrent.

In next sections we will cover the main components of LSPDC including preamplifier, post amplifier, inverse amplifier and the analogue to digital converter (ADC) in addition to the QD. And then we will discuss the implementation of the full position determination circuit with the detector. Also we will show how the microcontroller was used to get the output from the ADC, and perform the processing on the signal to calculate the position information by the program that shows the position graphically on the computer display.

\section{Laser Position Determination Circuit:}

Fig. 1 shows the general block diagram of the four quadrant detector with processing circuit for laser position determination. Starting from the output of the quadrant photodiode detector, the output of the photodiode is a current and with a very small value (with range in $\mu \mathrm{A}$ ), all the processing made on the signal treats the signal as a volt not as a current (due to that the used instruments and equipments work on the voltage). We can overcome this problem with the usage of the trance impedance (TI) amplifier which transfers the output current from the photodiode into equivalent value of the volt. The low level current can be raised by using the tranceimpedace amplifier (TIA) which is used to transfer and amplify the detector output current in to the volt. 


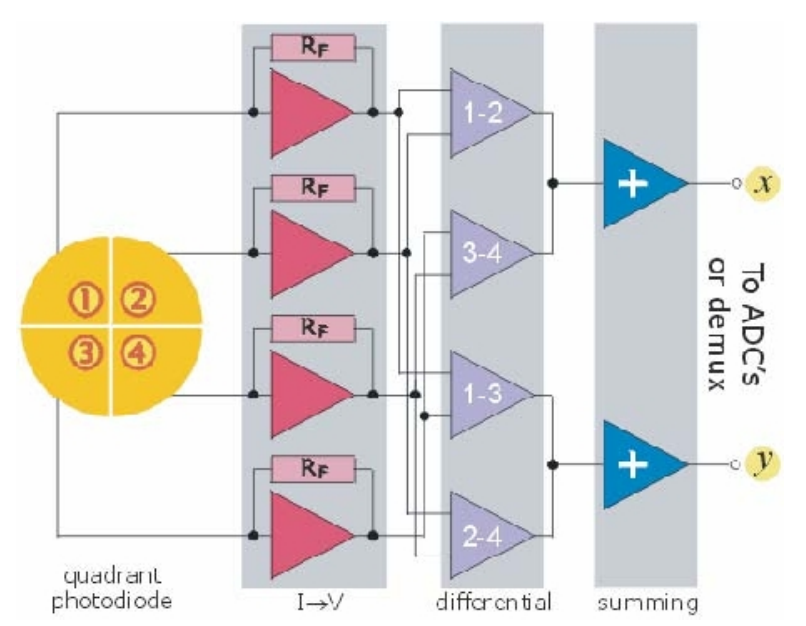

Figure (1): General block diagram for the 4 quadrant detector with processing circuit

First stage of Fig. 1 is the quadrant photodiode, in the second stage the photodiode output current is converted into the volt with the use of the TIA, the third stage is used to differentiate the four signals volts from each other, and in the last stage the summing of the signals is done to calculate the spot position, this is done using the following equations $[4,5,6]$ :

$$
\begin{aligned}
& X=K_{Q} \frac{\left(W_{1}+W_{3}\right)-\left(W_{2}+W_{4}\right)}{W_{1}+W_{2}+W_{3}+W_{4}} \\
& Y=K_{Q} \frac{\left(W_{1}+W_{2}\right)-\left(W_{3}+W_{4}\right)}{W_{1}+W_{2}+W_{3}+W_{4}}
\end{aligned}
$$

Where $\mathrm{X}$ and $\mathrm{Y}$ represent the position coordinate of the spot, 1,2,3,4 are the four signals from the four photodiodes as shown in Fig. 1, $\mathrm{K}$ is the proportional factor for the quadrant detector.

\section{Preamplifier Stage:}

Firstly we want to convert the photodiode output current to the volt this is used by the TIA. Preamplifier is the first stage in the system and is considered to be the most critical part. The integrating circuit has been used, which transforms current pulse into a voltage pulse with an amplitude proportional to a charge carried by the current pulse. In general, the function of a preamplifier is to amplify a low-level signal to the wanted level. The preamplifier provides voltage gain without significant current gain. The basic form of the TIA is the noninverting operational amplifier with the feedback resistance and capacitance as shown in Fig. 2 (a), the feedback resistance for the gain amplification value and the feedback capacitor is used to prevent the high frequency oscillation and noise, also act as a low pass filter [3]. 


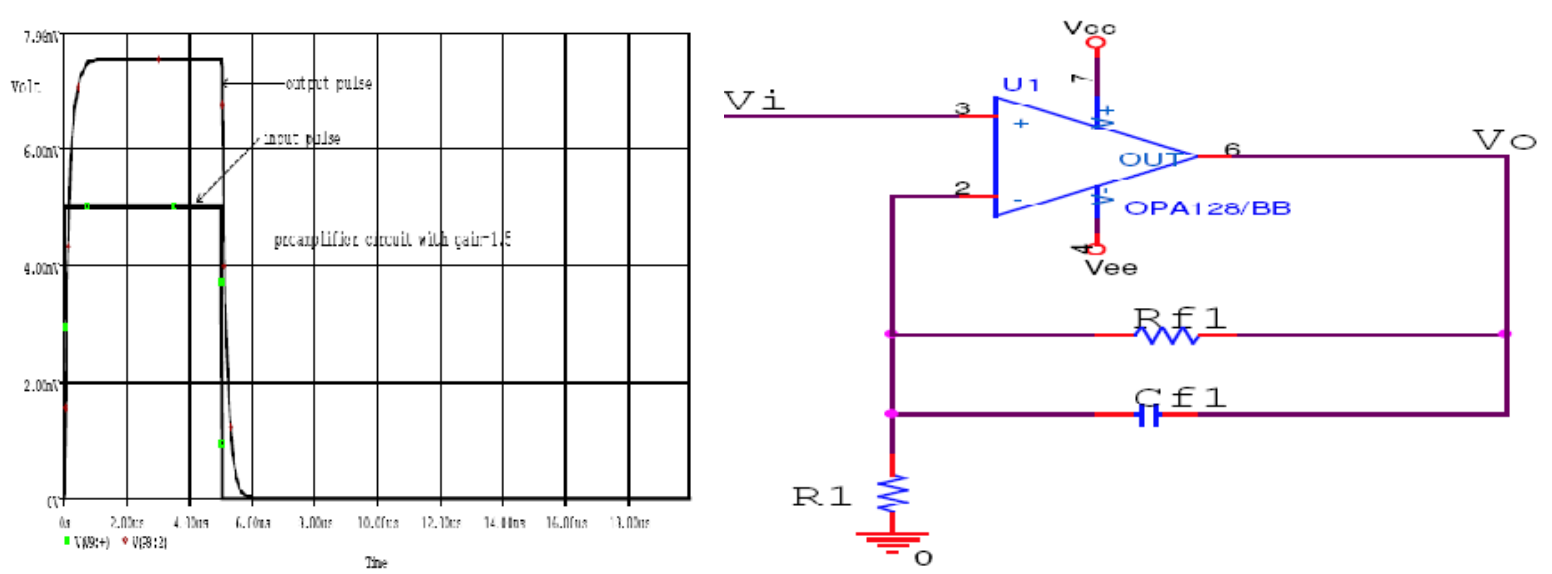

Figure (2): (a)General schematic of the non inverting operational (b) Outpot of the preamplifier with the input pulse.

From the circuit shown in Fig. 2 we can determine the gain of the circuit by the equation:

$$
\begin{aligned}
& \frac{V_{o}}{V_{i}}=G=\left(1+\frac{R_{f 1} / / C_{f 1}}{R_{1}}\right) \\
& G=\left(\begin{array}{c}
\frac{R_{f 1} / S C_{f 1}}{R_{f 1}+1 / S C_{f 1}} \\
R_{1}
\end{array}\right)=\left(1+\frac{R_{f 1} / S C_{f 1}}{R_{1} R_{f 1}+R_{1} / S C_{f 1}}\right) \\
& G=\left(1+\frac{R_{f 1}}{R_{1}+R_{1} R_{f 1} S C_{f 1}}\right)=\left(1+\frac{R_{f 1} / R_{1}}{1+R_{f 1} S C_{f 1}}\right)
\end{aligned}
$$

for the $\mathrm{DC}$ gain $\mathrm{S}=0$ so that the gain will be $G=\left(1+\frac{R_{f 1}}{R_{1}}\right)$

Figure (2-b) shows the calculated output of the preamplifier with the gain amplifier $\mathrm{G}=1.5$.

\section{Gain Circuit Stage:}

The second stage circuit (gain circuit) is used to increase the level of the output volt from the preamplifier because the preamplifier has a limited amplification level. By using the noninverting operational amplifier shown in Fig. (3-a), for the gain value determined according to the value of feedback resistance according to the basic equation of the operational amplifier [3]. We can determine the gain of the circuit by the equation: 


$$
\frac{V_{o 1}}{V_{o}}=\left(1+\frac{R_{f 2}}{R_{2}}\right)
$$
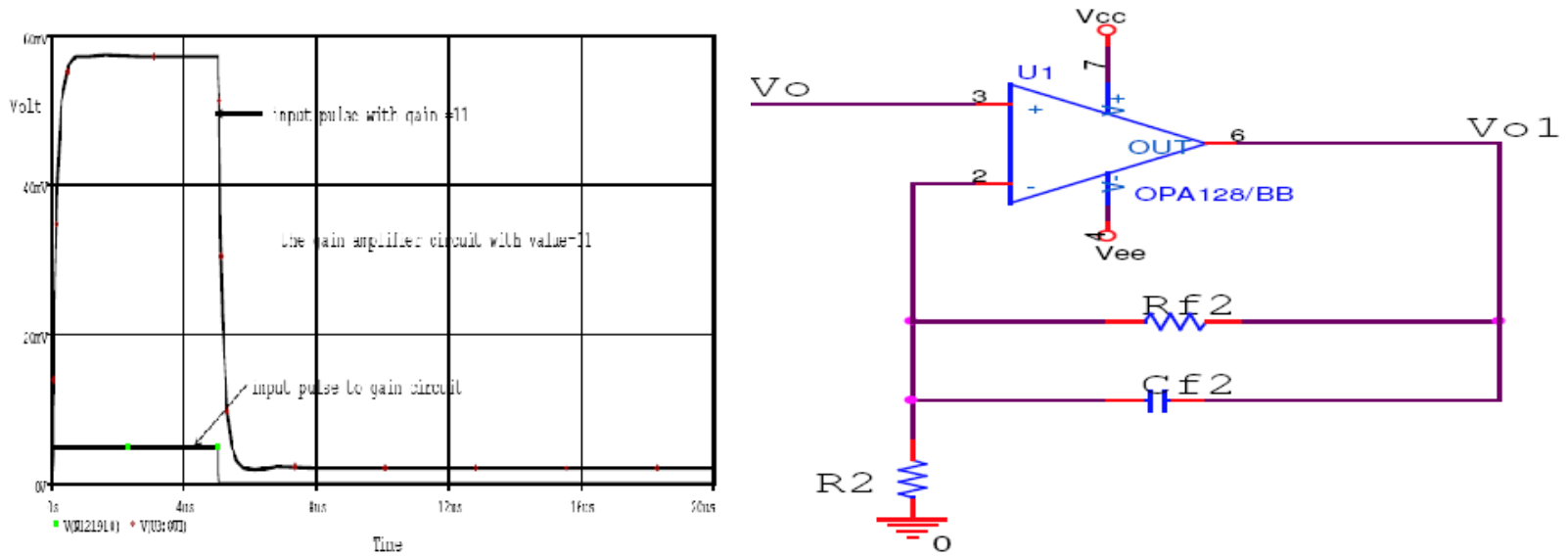

Figure (3): General schematic of the non inverting operational amplifier with the gain $(a)$, and $(b)$ the output of the gain amplifier with the input pulse.

by substituting from equation (5) into equation (7) we have to produce the equation which provide the summing of the two circuit as follows:

$V_{o 1}=V_{i}\left(1+\frac{R_{f 1} / R_{1}}{1+R_{f 1} S C_{f 1}}\right)\left(1+\frac{R_{f 2}}{R_{2}}\right)$

Figure (3-b) shows the output of the gain amplifier with the gain amplifier $\mathrm{G}=11$, the figure shows the two curves of the input with amplitude $(5 \mathrm{mv})$ and the output with amplitude $(55.56 \mathrm{mv})$ this prove the gain is $\mathrm{G}=11$.

\section{Inverse Signal Circuit Stage:}

The third stage is used for the inverse signal circuit (emitter follower) [3] with the gain value to be in positive sign and can be read with the analog to digital converter (ADC).
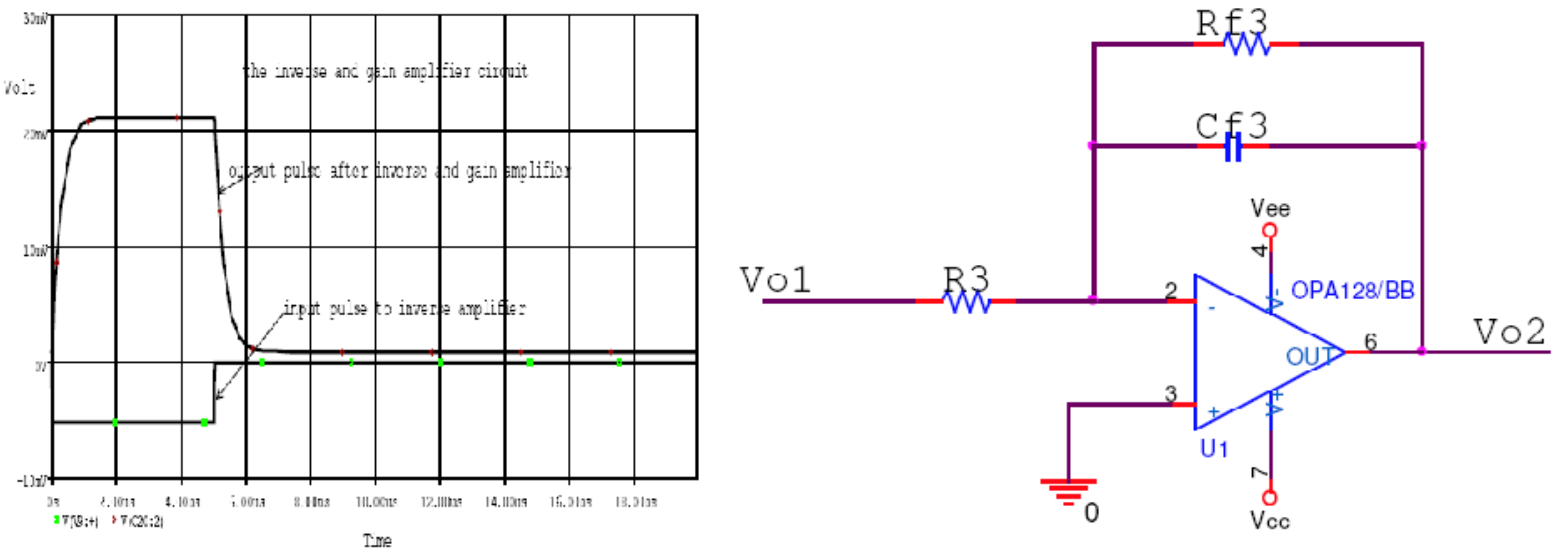

Figure (4): General schematic of the inverting operational amplifier with the gain 
amplitude (a), and (b) the output of the inverse amplifier with the input pulse.

From the circuit shown in Fig. (4-a) we can determine the gain of the circuit by the equation:

$$
\begin{aligned}
& \frac{V_{o 2}}{V_{o 1}}=\left(-\frac{R_{f 3} / / C_{f 3}}{R_{3}}\right) \\
& \frac{V_{o 2}}{V_{o 1}}=\left(-\frac{\frac{R_{f 3} / S C_{f 3}}{R_{f 3}+1 / S C_{f 3}}}{R_{3}}\right)=\left(-\frac{R_{f 3} / S C_{f 3}}{R_{3}\left(R_{f 3}+1 / S C_{f 3}\right)}\right) \\
& \frac{V_{o 2}}{V_{o 1}}=\left(-\frac{R_{f 3}}{\left.R_{3} R_{f 3} S C_{f 3}+R_{3}\right)}\right)
\end{aligned}
$$

For the $\mathrm{DC}$ gain $\mathrm{S}=0$ so that the gain will be

$\frac{V_{o 2}}{V_{o 1}}=\left(-\frac{R_{f 3}}{\left.R_{3}\right)}\right)$

by substituting from equation (8) into equation (11) we have to produce the equation which provide the summing of the two circuits as follows:

$$
V_{o 2}=-V_{i}\left(1+\frac{R_{f 1} / R_{1}}{1+R_{f 1} S C_{f 1}}\right)\left(1+\frac{R_{f 2}}{R_{2}}\right)\left(\frac{R_{f 3}}{R_{3}+R_{3} R_{f 3} S C_{f 3}}\right)
$$

for the $\mathrm{DC}$ gain $\mathrm{S}=0$ so that the gain will be the total system amplification given by:

$$
V_{o 2}=-V_{i}\left(1+\frac{R_{f 1}}{1+R_{1}}\right)\left(1+\frac{R_{f 2}}{R_{2}}\right)\left(\frac{R_{f 3}}{R_{3}}\right)
$$

Figure (4-b) shows the output of the inverse amplifier with the gain amplifier, the figure shows the two curves of the input and the output after inverse and gain amplified.
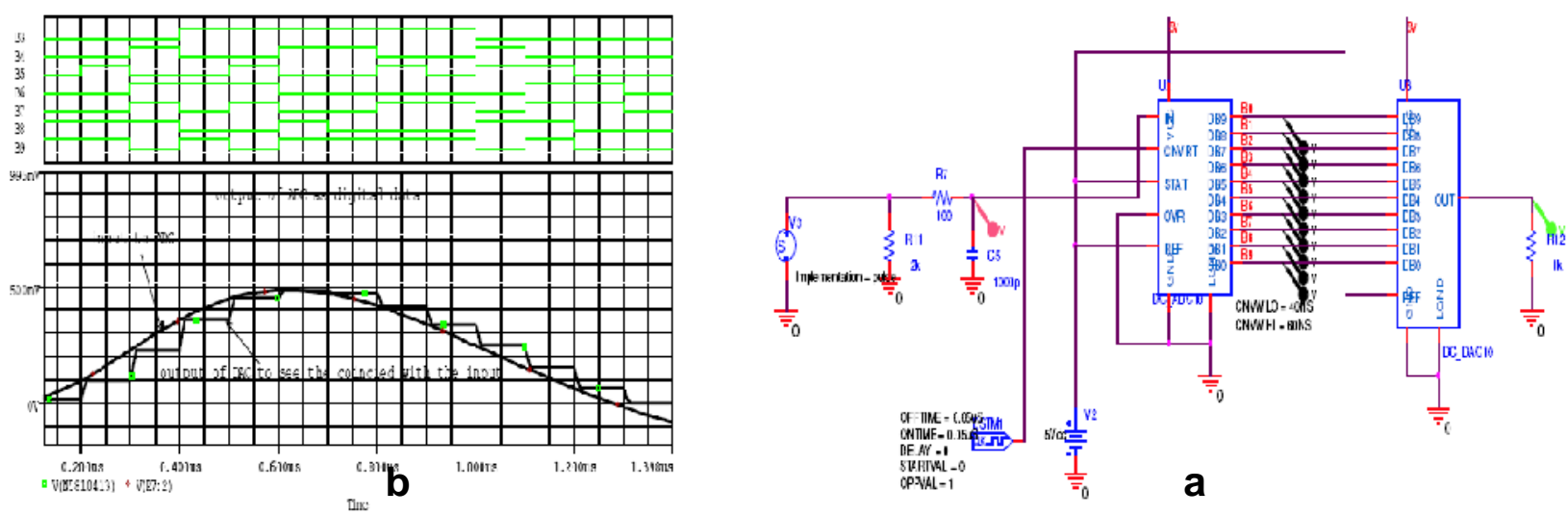

Figure (5): Schematic diagram of the analog to digital converter $(A D C)$ and the digital to analogue converter (a), (b) input pulse to the ADC and the digital output of the ADC.

\section{Analog to Digital Converter Stage:}


Figure (5-a) shows the circuit diagram of the analog to digital converter (ADC) [3] and the digital to analog converter (DAC) to make sure that the input to ADC is the same as the output of the DAC . Now the output at the Vo2 will be the input to the ADC to take the value of each part of the quadrant photodiode and take the output from the analog to digital converter into the microcontroller to apply the processing on the output signal and make the determination of the laser spot position. Figure (5-b) shows the input and the output of the analog to digital converter (ADC) and the digital to analog converter to make sure that the input to ADC is the same as the output of the DAC, from the figure it is clear that the input to the ADC have a good agreement with the output of the DAC.

\section{Laser Spot Position Determination Circuit Diagram:}

Now we will use the full circuit diagram for the laser spot position determination. Starting with the photodiode model $[1,2]$ and including the preamplifier circuit, the gain circuit, the inverse amplifier, and the ADC. The analogue signal output from each section of the 4 quadrant detector is to be read from the microcontroller to make the processing on the signal and provide $\mathrm{X}$ and $\mathrm{Y}$ position of the laser spot.

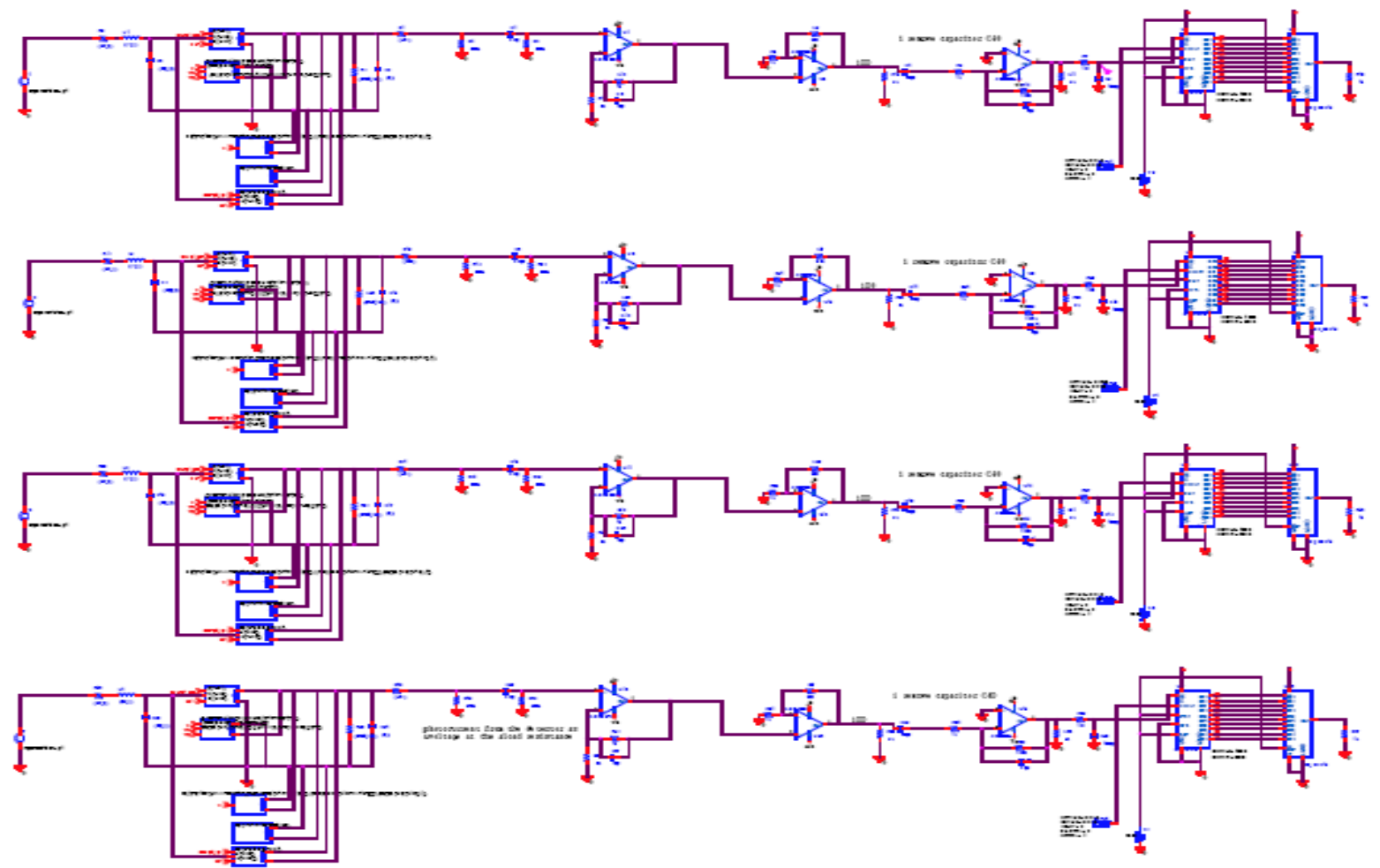

Figure (6): the full schematic of the laser spot position determination circuit.

Figure (6) shows the full schematic of the laser spot position determination circuit [1,2].

\section{System Simulation:}

To examine the system model performance, we will show the simulation of different spot position on the quadrant detector. 
Figure (7-a) shows the laser spot with the quadrant detector when the laser spot at the center of the quadrant detector. When the spot is at the center of the quadrant detector the illuminated areas of the four photodiode are the same, then the represented value for the incident radiation is the same for the four photodiodes.
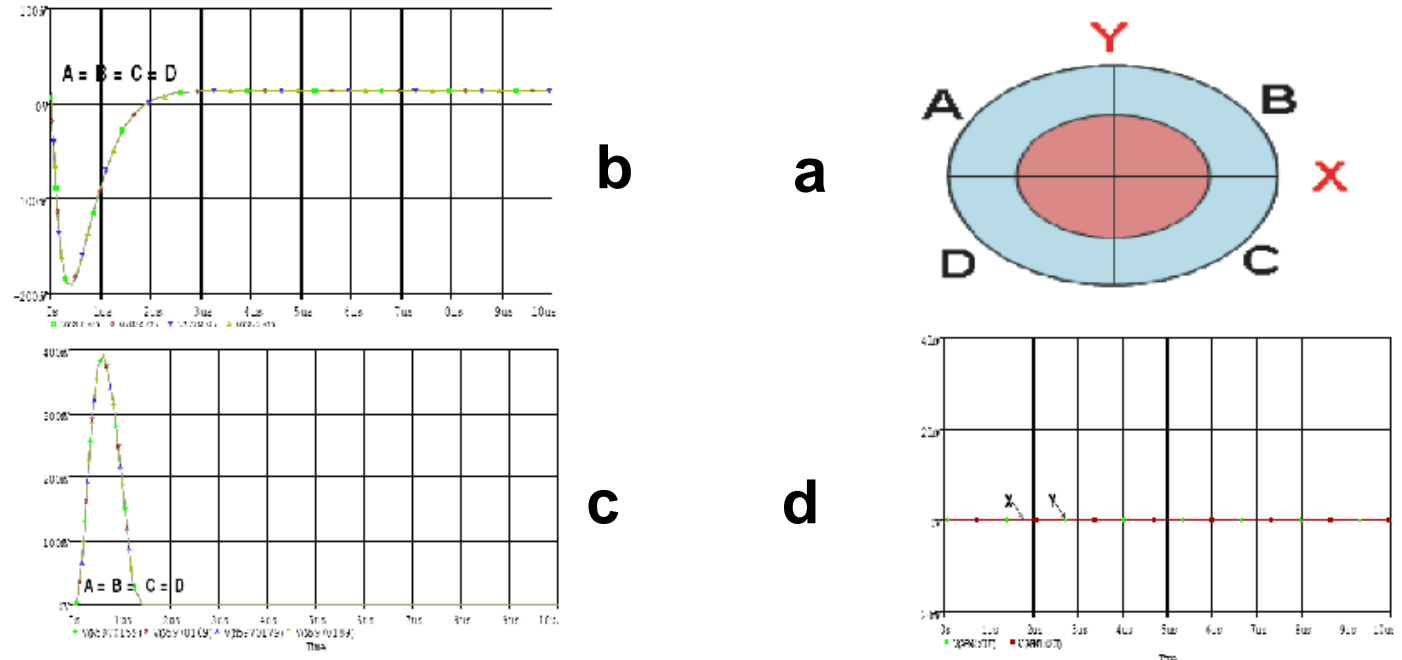

Figure (7): laser spot at the center of $Q D(a), 4 P D$ output after amplifier (b), after inverse amplifier (c), spot position interims of $X$ and $Y(d)$.

Figure (7-b) shows the output of the four photodiode after the amplifier, the output is the same. Figure (7-c) shows the output of the four photodiode after the inverse amplifier, the positive output is needed since the ADC works on the positive signal, for the position information we take the output from ADC and make processing on it to calculate the $\mathrm{Y}$ and $\mathrm{X}$ equations for laser spot determination according to equation (1) and (2). Figure (7-d) shows the output value of $X$ and $Y$ for this condition $X=Y=0$ due to spot center with the detector center. Second case for the laser spot shifted in $\mathrm{Y}$ axis and zero shift in $\mathrm{X}$ axis the incident radiation on $\mathrm{C}=\mathrm{D}=2 \mathrm{~A}=2 \mathrm{~B}$. 

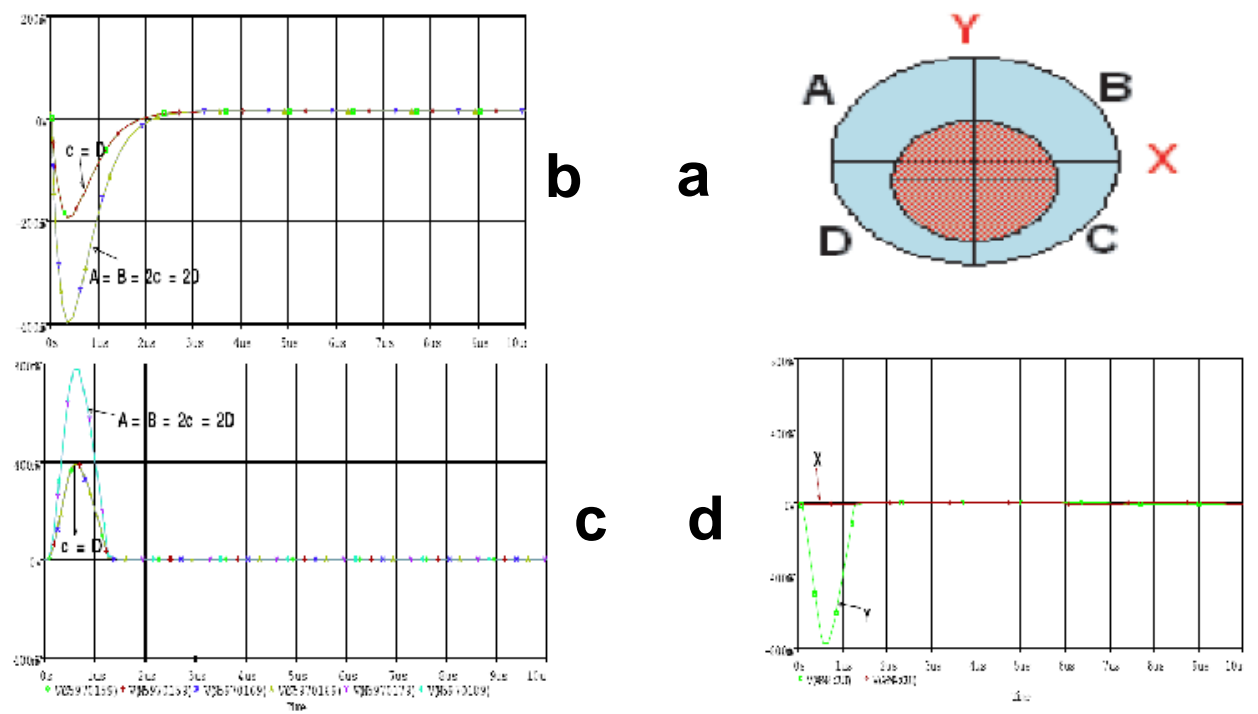

Figure (8): laser spot shifted from the center of the quadrant detector (a), 4 PD output after amplifier (b), after inverse amplifier (c), spot position interims of $X$ and $Y(d)$. Figure (8-a) show the laser spot with the quadrant detector the laser spot shifted from the center of the quadrant detector in negative $\mathrm{Y}$ axis and zero shift in $\mathrm{X}$ axis Starting with the spot position shifted in $\mathrm{Y}$ axis from the center of the quadrant detector in this case the illuminated areas of the four photodiode are not the same $(A=B, C=D)$, then the represented value for the incident radiation according to the illuminating area of every photodiode. Figure (8-b) show the output of the four photodiode after the amplifier where the output of A and B is the same and the output of C and D is the same due to the incident radiation on the photodiode $A=B$ and $C=D$. Figure (8-c) show the output of the four photodiode after the inverse amplifier, the positive output due to the ADC work on the positive signal. for the position information we take the output from $\mathrm{ADC}$ and make processing on it to calculate the $\mathrm{Y}$ and $\mathrm{X}$ equations for laser spot determination according to equation (1) and (2). Figure (8-d) show the output value of $X$ and $\mathrm{Y}$ for this condition $\mathrm{X}=0$ and $\mathrm{Y}=-\mathrm{ve}$ value due to spot center shifted in the negative $\mathrm{Y}$ with the detector center.

Third case for the laser spot shifted in $\mathrm{X}$ axis and zero shift in $\mathrm{Y}$ axis the incident radiation in this case $\mathrm{B}=\mathrm{C}=2 \mathrm{~A}=2 \mathrm{D}$. 

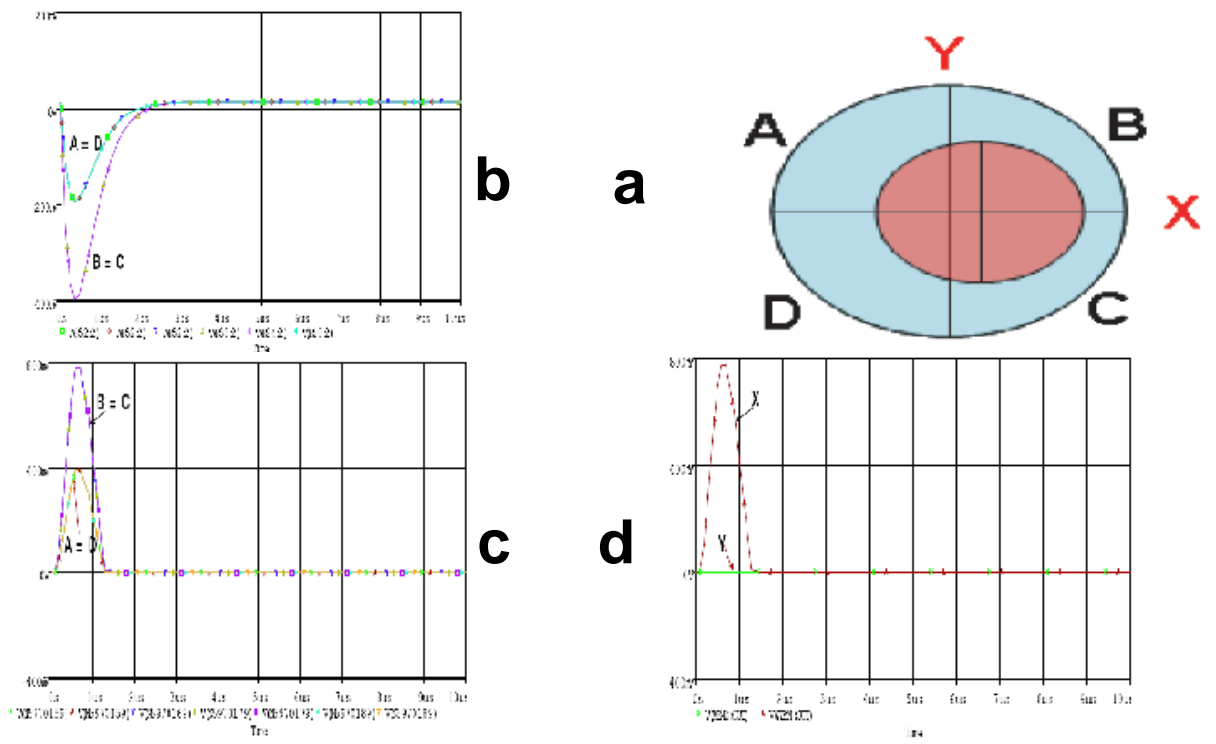

Figure (9): laser spot shifted from the center of the quadrant detector (a), 4 PD output after amplifier (b), after inverse amplifier (c), spot position interims of $X$ and $Y(d)$.

Figure (9-a) show the laser spot with the quadrant detector the laser spot shifted from the center of the quadrant detector in positive $\mathrm{X}$ axis and zero shift in $\mathrm{Y}$ axis Starting with the spot shifted in $\mathrm{X}$ axis from the center of the quadrant detector in this case the illuminated areas of the four photodiodes are not the same and follow the condition $\mathrm{B}=\mathrm{C}=2 \mathrm{~A}=2 \mathrm{D}$, then the represented value for the incident radiation is not the same for the four photodiodes. Figure (9-b) shows the output of the four photodiode after the amplifier the output of B and C is the same and the output of A and D is the same due to the incident radiation on the photodiode $\mathrm{B}=\mathrm{C}$ and $\mathrm{A}=\mathrm{D}$. Figure (9-c) shows the output of the four photodiode after the inverse amplifier, the positive output due to the ADC work on the positive signal. Figure (9-d) shows the output value of $\mathrm{X}$ and $\mathrm{Y}$ for this condition $Y=0$ and $X=+v e$ value due to spot center shifted in the positive $X$ with the detector center. Forth case for the laser spot shifted in $\mathrm{X}$ axis and shift in $\mathrm{Y}$ axis the incident radiation on $\mathrm{A}=2 \mathrm{~B}=2 \mathrm{C}=2 \mathrm{D}$. 

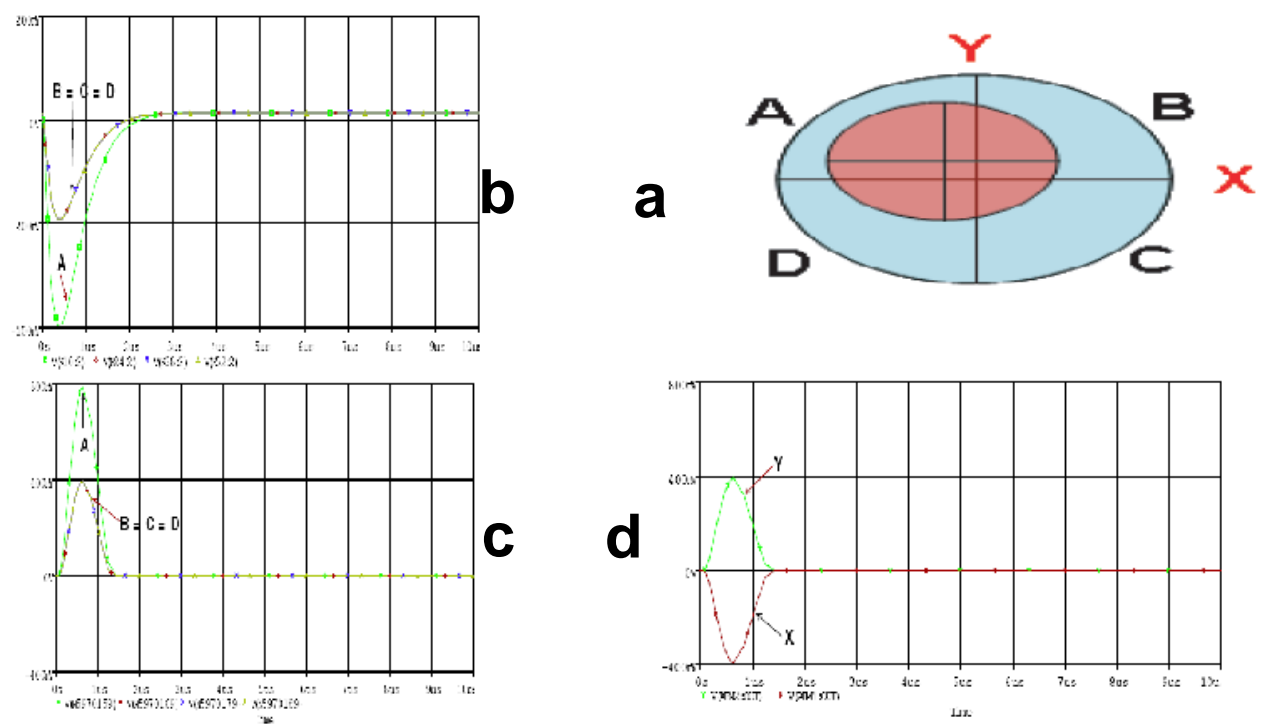

Figure (10): laser spot shifted from the center of the quadrant detector, 4 PD output after amplifier (b), after inverse amplifier (c), spot position interims of $X$ and $Y(d)$.

Figure (10-a) shows the laser spot with the quadrant detector the laser spot shifted from the center of the quadrant detector in positive $\mathrm{X}$ axis and zero shift in $\mathrm{Y}$ axis. Starting with the spot shifted in $\mathrm{X}$ axis from the center of the quadrant detector in this case the illuminated areas of the four photodiodes are not the same and follow the condition $\mathrm{B}=\mathrm{C}=2 \mathrm{~A}=2 \mathrm{D}$, then the represented vale for the incident radiation is not the same for the four photodiodes. Figure (10-b) shows the output of the four photodiode after the amplifier the output of $\mathrm{B}$ and $\mathrm{C}$ is the same and the output of $\mathrm{A}$ and $\mathrm{D}$ is the same due to the incident radiation on the photodiode $\mathrm{B}=\mathrm{C}$ and $\mathrm{A}=\mathrm{D}$. Figure (10-c) shows the output of the four photodiode after the inverse amplifier, the positive output due to the ADC work on the positive signal. for the position information we take the output from ADC and make processing on it to calculate the $\mathrm{Y}$ and $\mathrm{X}$ equations for laser spot determination according to equation (1) and (2). Figure (10-d) shows the output value of $\mathrm{X}$ and $\mathrm{Y}$ for this condition $\mathrm{X}=$-ve and $\mathrm{Y}=+\mathrm{ve}$ value due to spot center shifted in the negative $\mathrm{X}$ and $+\mathrm{ve} \mathrm{Y}$ axis with the detector center.

We conclude that according to the value of incident radiation and the illuminating areas of photodiode the center of the spot can be evaluated with respect to quadrant detector center.

\section{PCB Design For LSPDC:}

Figure (11) shows the routing fill and the designed PCB card and also the distribution of the components on the two cards which contain the schematic from the preamplifier to the ADC. 


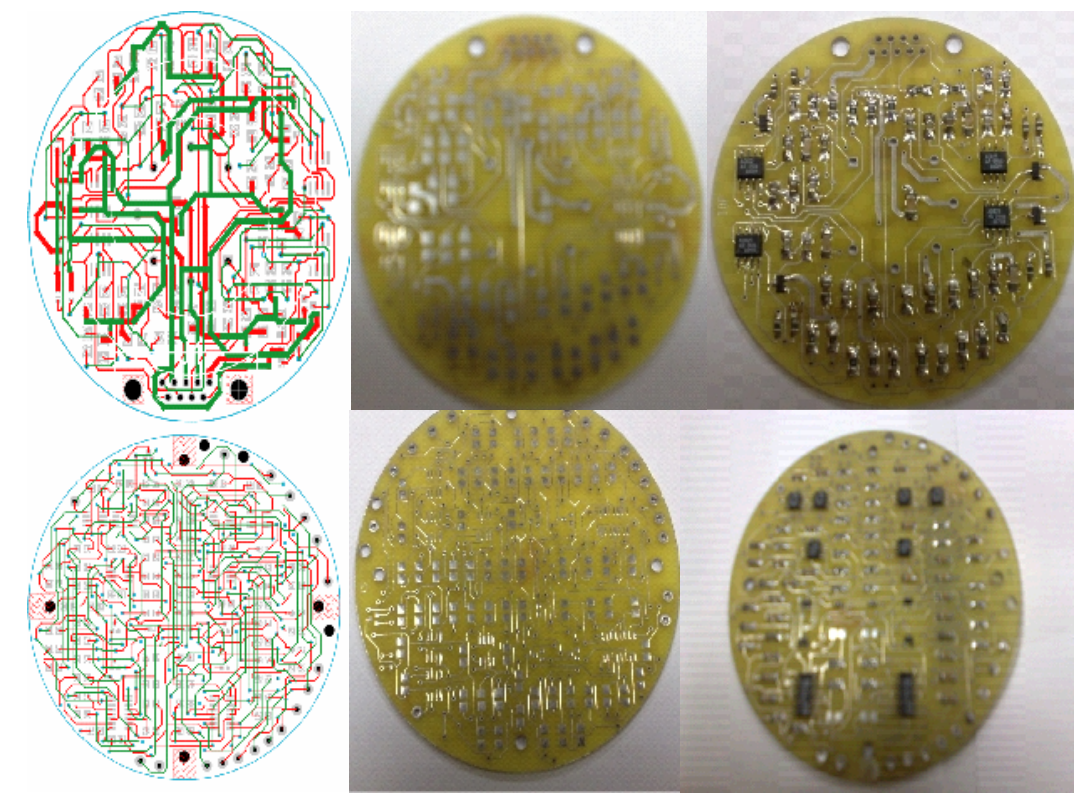

Figure (11): Routing fill, PCB design, component distribution

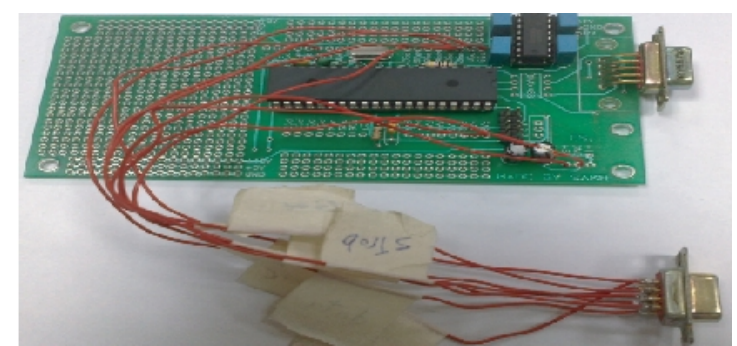

Figure (12): Microcontroller card used to make processing on the data output from $A D C$.

Figure (12) shows the microcontroller card that used to perform processing on the data output from ADC. A microcontroller was used to perform processing on the signal and calculate the spot position on the detector.

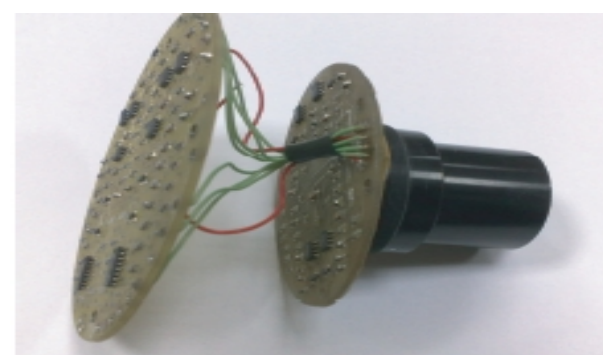

Figure (13): Assembled system with the necessary optical system.

\section{System Measurements:}

Figure (14) shows the general block diagram for laser spot position determination system containing laser source that simulating the incident radiation, optical system to collimate incident radiation on the quadrant detector, microcontroller to make 
processing and calculation, the mechanical system to adjust seeker at different angles.

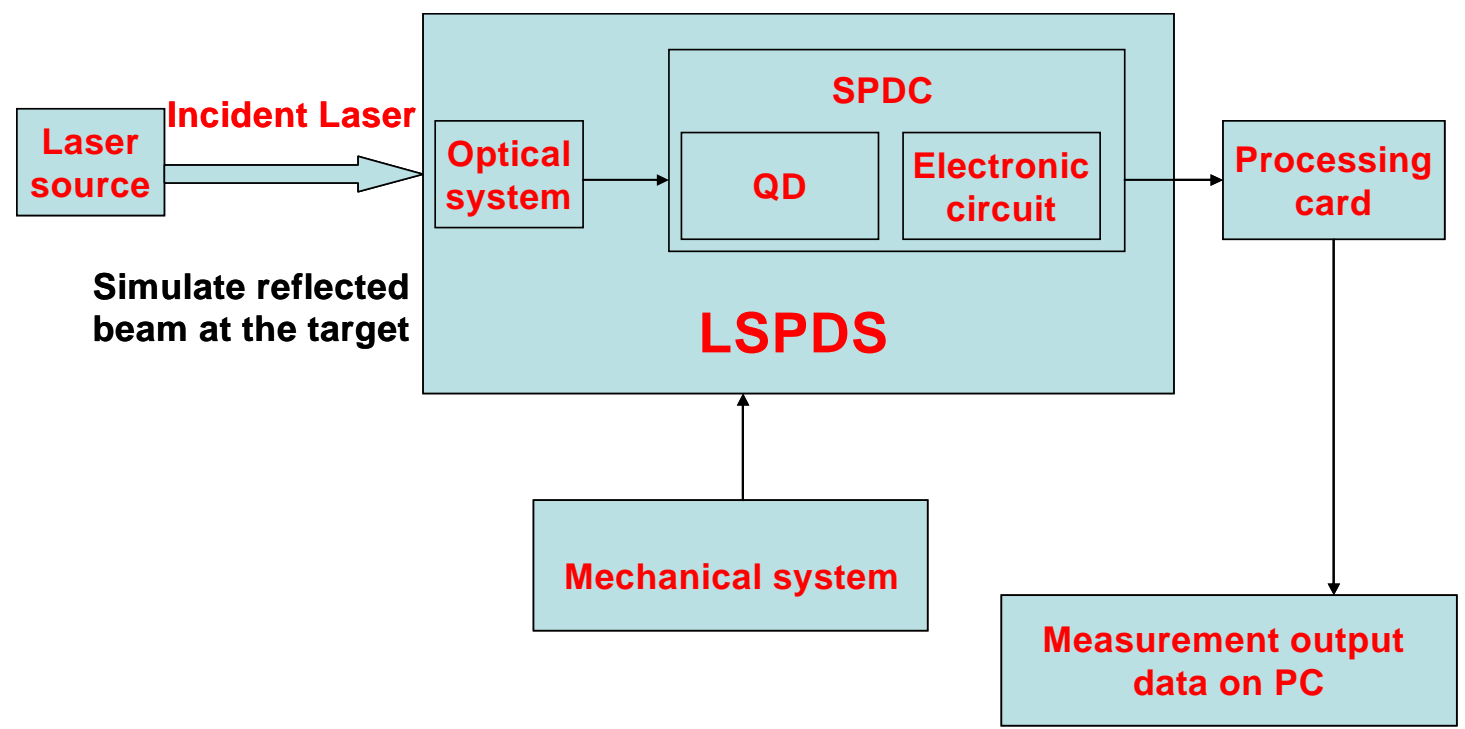

Figure (14): General block diagram for the laser spot position determination measurement.

Figure (15) shows the complete system including the microcontroller card for the processing on the output signal to determine the spot position. A microcontroller was used to perform processing on the signal and calculate the spot position on the detector. We put the seeker on the moving mechanical system to be adjusted at different angles in the direction of laser, with the help of the processed program we calculated the spot position on the detector as shown below:

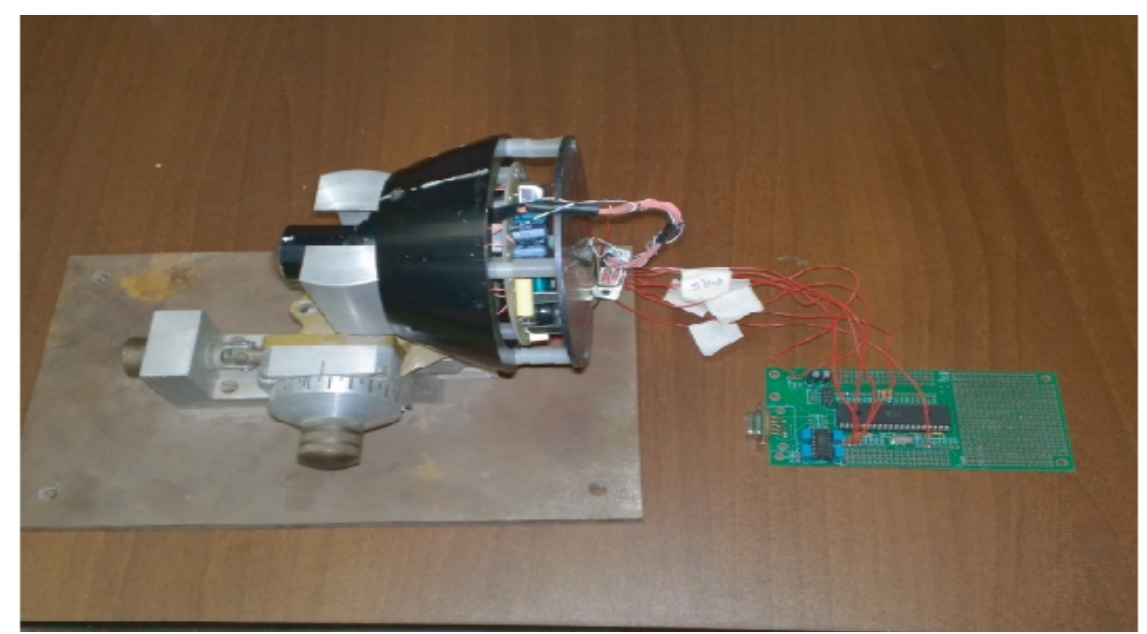

Figure (15): Complete system including the microcontroller card . 

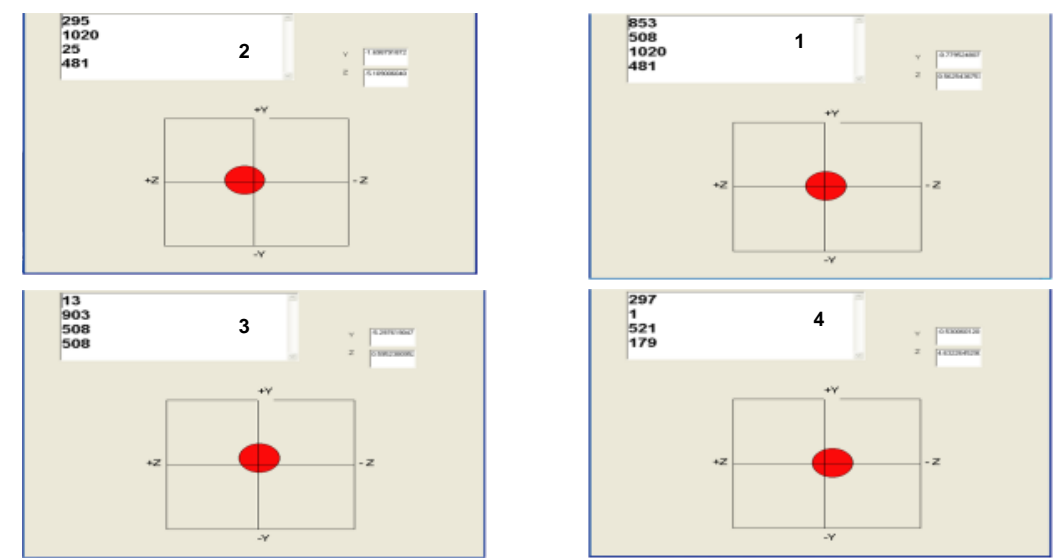

Figure (16): Calculation of the spot position at the detector with the seeker adjusted at different angle of $Z$ and $Y$.

Figure (16.1) shows the calculation of the spot position at the detector with the seeker adjusted at $Y=0$ and $Z=0$. From figure (16.1) we conclude that when the seeker positioned at $Y=0$ degree and $Z=0$ degree, the measurement of the spot on the detector $Y=0.7$ degree and $Z=0.5$ degree has a good agreement with the seeker position and the small difference value is due to the error in the mechanical system and the stability of the available laser source. Figure (16.2) shows the calculation of the spot position at the detector with the seeker adjusted at $Y=0$ and $Z=5$. From figure (16.2) we conclude that when the seeker positioned at $Y=0$ degree and $Z=5$ degree, the measurement of the spot on the detector $\mathrm{Y}=0.5$ degree and $\mathrm{Z}=4.63$ degree has a good agreement with the seeker position and the small difference value is due to the error in the mechanical system and the stability of the available laser source. Figure (16.3) shows the calculation of the spot position at the detector with the seeker adjusted at $Y=5$ degree and $Z=0$ degree. From figure (16.3) we conclude that when the seeker positioned at $Y=5$ degree and $Z=0$ degree, the measurement of the spot on the detector $Y=5.29$ degree and $Z=0.59$ degree has a good agreement with the seeker position and the small difference value is due to the error in the mechanical system and the stability of the available laser source. Figure (16.4) shows the calculation of the spot position at the detector with the seeker adjusted at $Y=0$ degree and $Z=-5$ degree. From figure (16.4) we conclude that when the seeker positioned at $\mathrm{Y}=0$ degree and $\mathrm{Z}=-5$ degree the measurement of the spot on the detector $\mathrm{Y}=0.5$ degree and $\mathrm{Z}=-4.63$ degree has a good agreement with the seeker position and the small difference value is due to the error in the mechanical system and the stability of the available laser source. 

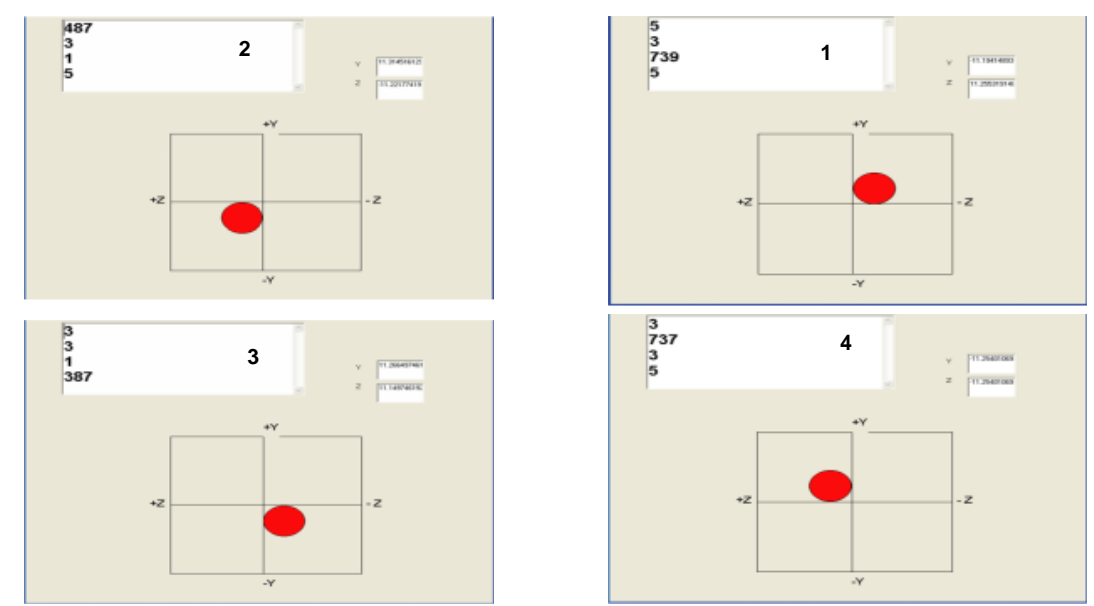

Figure (17): Calculation of the spot position at the detector with the seeker adjusted at different angle of $Z$ and $Y$.

Figure (17.1) shows the calculation of the spot position at the detector with the seeker adjusted at $Y=10$ degree and $Z=-10$ degree. From figure (17.1) we conclude that when the seeker positioned at $Y=10$ degree and $Z=-10$ degree, the measurement of the spot on the detector $\mathrm{Y}=11.2$ degree and $\mathrm{Z}=-11.2$ degree has a good agreement with the seeker position and the small difference value is due to the error in the mechanical system and the stability of the available laser source. Figure (17.2) shows the calculation of the spot position at the detector with the seeker adjusted at $Y=-10$ degree and $Z=10$ degree. From figure (17.2) we conclude that when the seeker positioned at $\mathrm{Y}=-10$ degree and $\mathrm{Z}=10$ degree the measurement of the spot on the detector $\mathrm{Y}=-11.3$ degree and $\mathrm{Z}=11.2$ degree, has a good agreement with the seeker position and the small difference value is due to the error in the mechanical system and the stability of the available laser source. Figure (17.3) shows the calculation of the spot position at the detector with the seeker adjusted at $Y=-10$ degree and $Z=-10$ degree. From figure (17.3) we conclude that when the seeker positioned at $Y=-10$ degree and $\mathrm{Z}=-10$ degree, the measurement of the spot on the detector $\mathrm{Y}=-11.26$ degree and $\mathrm{Z}=-$ 11.2 degree has a good agreement with the seeker position and the small difference value is due to the error in the mechanical system and the stability of the available laser source. Figure (17.4) shows the calculation of the spot position at the detector with the seeker adjusted at $Y=10$ degree and $Z=10$ degree. From figure (17.4) we conclude that when the seeker positioned at $Y=10$ degree and $Z=10$ degree the measurement of the spot on the detector $Y=11.2$ degree and $Z=11.2$ degree, has a good agreement with the seeker position and the small difference value is due to the error in the mechanical system and the stability of the available laser source. 

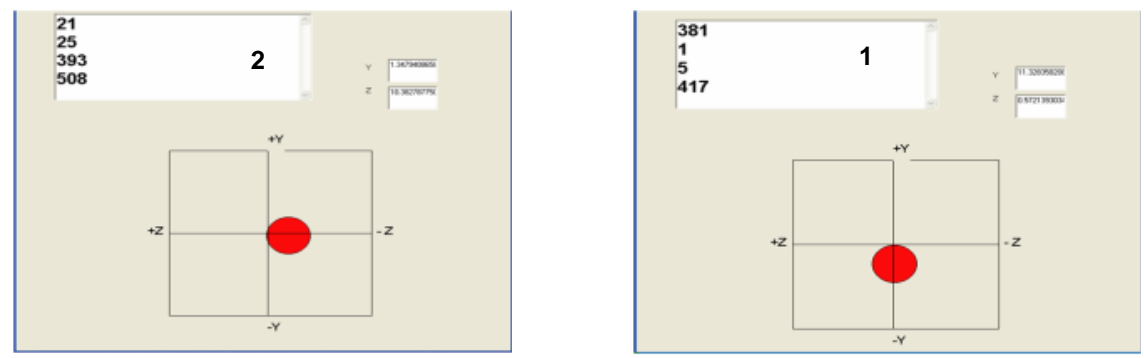

Figure (18): The calculation of the spot position at the detector with the seeker adjusted at different angle of $Z$ and $Y$.

Figure (18.1) shows the calculation of the spot position at the detector with the seeker adjusted at $Y=-10$ degree and $Z=0$ degree. From figure (18.1) we conclude that when the seeker positioned at $Y=-10$ degree and $Z=0$ degree, the measurement of the spot on the detector $Y=-11.3$ degree and $Z=0.57$ degree has a good agreement with the seeker position and the small difference value is due to the error in the mechanical system and the stability of the available laser source. Figure (18.2) shows the calculation of the spot position at the detector with the seeker adjusted at $Y=0$ degree and $Z=-10$ degree. From figure (18.2) we conclude that when the seeker positioned at $Y=0$ degree and $Z=-10$ degree, the measurement of the spot on the detector $Y=1.3$ degree and $Z=-10.4$ degree has a good agreement with the seeker position and the small difference value is due to the error in the mechanical system and the stability of the available laser source.

Figures $16-18$ an be summarized in table 1 .

Table (1): The adjusted and measurement data of the detector position .

\begin{tabular}{|c|c|c|c|c|c|c|}
\hline angle (degree) & adjusted Y & adjusted Z & measured Y & measured Z & Y error & $\mathrm{Z}$ error \\
\hline 0 & 0 & 0 & 0.7 & 0.5 & 0.7 & 0.5 \\
5 & 0 & 5 & 0.5 & 4.63 & 0.5 & 0.37 \\
5 & 5 & 0 & 5.29 & 0.59 & 0.29 & 0.59 \\
5 & 0 & -5 & 0.5 & -4.63 & 0.5 & -0.37 \\
5 & 5 & 5 & 4.9 & 6.1 & 0.1 & 1.1 \\
10 & 10 & 10 & 11.2 & 11.2 & 1.2 & 1.2 \\
10 & -10 & -10 & -11.26 & -11.2 & -1.2 & -1.2 \\
10 & 10 & -10 & 11.2 & -11.2 & 1.2 & -1.2 \\
10 & -10 & 10 & -11.3 & 11.2 & -1.2 & 1.2 \\
10 & 0 & -10 & 1.3 & 10.4 & 1.3 & -0.4 \\
10 & -10 & 0 & 11.3 & 0.57 & -1.3 & 0.57 \\
\hline
\end{tabular}

We conclude that at any change of the seeker position the spot position on the detector changes and from all figures the spot coincides with the measurement ranging from $93 \%$ to $97 \%$. The error in value is attributed to the error in the moving mechanical system used to adjust seeker to different angles and the effect of the field of view on the optical system.

\section{Conclusions:}

LSPDC including QD, preamplifier, post amplifier, and ADC was analyzed and modeled. The LSPDC was implemented in ORCAD pspise tool and then implemented in hardware. The validation of the model using a proposed setup was performed using a laser source simulating the incident radiation and a mechanical system to enable 
adjusting different angles; good agreement was achieved between the measured and simulated results.

\section{References:}

[1] Mohamed F. Heweage, Ayman M Mokhtar, Mekheimer A. Soliman, Modeling and Performance Analysis of a Quad Detector,ASAT-14,2011.

[2] R. J. Perry and K. Arora, IUsing pspice to simulate the photoresponse of ideal cmos integrated circuit photodiodes," IEEE, 1996.

[3] http://en.wikipedia.org/wiki/Operational_amplifier_applications.

[4] Z. P. Barbaric1 and L. M. Manojlovic, IOptimization of optical receiver parameters for pulsed laser tracking systems," Microwave Review, September 2003.

[5] L. G. Kazovsky, ITheory of tracking accuracy of laser systems," Optical Engineering, vol. 22, no. 3, pp. 339\{347, May/June 1983.

[6] E. O. Doebelin, IMeasurement systems application and design," Mc Graw-Hill, 1990.

[7] K. A. Masahiro Toyoda and Y. Suzuki, IMeasurement of the characteristics of a quadrant avalanche photodiode and its application to a laser tracking system," Optical Engineering,, vol. 41, no. 1, January 2002. 